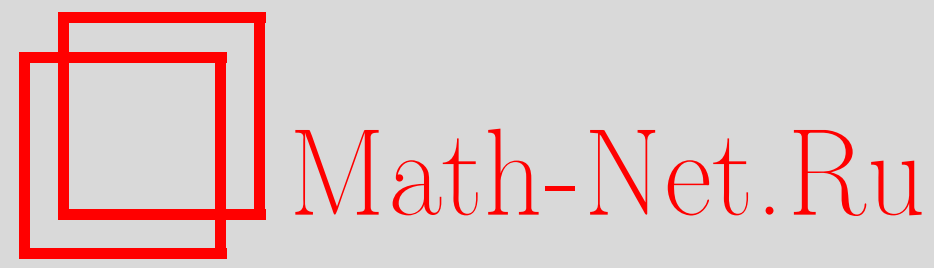

Г. В. Балакин, О структуре решений системы из линейных псевдобулевых неравенств, Матем. вопр. криптогр., 2012, том 3, выпуск 3, 5-19

DOI: https://doi.org/10.4213/mvk58

Использование Общероссийского математического портала Math-Net.Ru подразумевает, что вы прочитали и согласны с пользовательским соглашением

http://www.mathnet.ru/rus/agreement

Параметры загрузки:

IP : 54.166 .219 .16

26 апреля 2023 г., 06:20:44 
УДК: 519.212.2

\title{
О структуре решений системы из линейных псевдобулевых неравенств
}

\author{
Г. В. Балакин \\ Академия криптографии Российской Федерации, Москва
}

Получено 20.V.2011

В работе рассматриваются случайные и заведомо совместные системы из линейных псевдобулевых неравенств. Для случайных систем найдены алгебраические условия совместности, а для заведомо совместных систем найдено среднее число решений, отличающихся от истинного в двух координатах.

Ключевые слова: пороговые функции, линейные псевдобулевы неравенства

On the structure of solutions of nonlinear pseudo-Boolean inequalities systems

\author{
G. V. Balakin \\ Academy of Cryptography of Russian Fereration, Moscow
}

Abstract. Random and random satisfiable systems of linear pseudo-Boolean inequalities are considered. For random systems we find algebraic conditions of satisfiability; for random satisfiable systems we find the mean number of solutions which differ from the true solution by 2 coordinates only.

Key words: threshold functions, linear pseudo-Boolean inequalities

Citation: Mathematical Aspects of Cryptography, 2012, vol. 3, no. 3, pp. 5-19 (Russian).

(C) 2012 Г. В. Балакин 


\section{§1. Введение}

Как известно, линейная булевая функция, существенно зависящая от $n$ переменных, отличается от нелинейной только одним свойством: вершинам на каждом ребре $n$-мерного единичного куба приписываются разные значения функции. Таким образом, на вершинах $n$-мерного единичного куба возникает как бы решетка из чередующихся нулей и единиц. Если на этой решетке хотя бы на одном ребре соседствуют два одинаковых значения, а соответствующая функция существенно зависит от всех переменных, то эта функция нелинейная. По существу, такие события определяют ошибки при замене этой функции линейным булевым аналогом.

Для нелинейных функций характерно наличие связных областей вершин с одинаковыми значениями функции в них. Создается впечатление, что чем меньше таких областей и чем больше вершин в них, тем сильнее функция, близкая к равновероятной, отличается от линейной, и ее нецелесообразно заменять на линейный булев аналог. В этом плане представляется, что худшей функцией будет пороговая функция. Действительно, пороговая функция характеризуется двумя связными областями, которые можно разделить одной плоскостью (линейным псевдобулевым неравенством). Поэтому не имеет смысла заменять такие функции и функции, близкие к ним, линейными булевыми аналогами.

В связи с этим возникает интерес к замене нелинейных булевых уравнений линейными псевдобулевыми неравенствами (пороговыми функциями), возможно, и с ошибками $[1,2]$. Но вначале надо указать, какие псевдобулевы линейные неравенства мы будем рассматривать. Во-первых, надо определиться со значениями коэффициентов в этих неравенствах, а во-вторых, со значениями правых частей и знаков неравенств.

\section{§2. Объект исследования}

Будем считать, что коэффициенты в псевдобулевых неравенствах с $n$ неизвестными принимают только два значения: +1 или -1 . Всего возможных левых частей неравенств $2^{n}$. По существу дела предлагается булево уравнение (функцию) заменять ее статистическим аналогом - псевдобулевым неравенством (пороговой функцией). Причем на пороговую функцию (неравенство) накладываются приведенные выше ограничения.

Изучению таких неравенств посвящены работы [3-5]. В этих работах проведен предварительный анализ случайных систем неравенств $(\mathrm{CCH})$ и заведомо совместных случайных систем неравенств (ЗСССН). В частности, 
найдены условия, при выполнении которых $\mathrm{CCH}$ не имеет решений, а также оценена возможность применения метода выделения и оценки отдельных неизвестных для нахождения решений ЗСССН.

Не теряя общности, можно предположить, что $n$ - четное число, $\left|x^{0}\right|=x_{1}^{0}+\ldots+x_{n}^{0}=m$ - нечетное число, $x^{0}=\left(x_{1}^{0}, \ldots, x_{n}^{0}\right)$ - истинное peшение 3СССН. Коэффициенты в псевдобулевых неравенствах ССН и ЗСССН являются реализациями случайных величин, принимающих только два значения $(+1$ или -1$)$ с равными вероятностями. Назовем приведенные условия случаем $\mathcal{A}$, а соответствующий класс пороговых функций обозначим $\mathcal{K}$.

В случае $\mathcal{A}$ левая часть неравенств исходной системы принимает только нечетные значения для наборов неизвестных нечетного веса. Неравенства со знаком $\leq$ преобразуем в неравенства со знаком $\geq$. В приведенной системе будут присутствовать только неравенства вида

$$
a_{i 1} x_{1}+a_{i 2} x_{2}+\ldots+a_{i n} x_{n} \geq 1 \text {. }
$$

Число различных неравенств вида (1) для ССH равно $2^{n}$, так как знак неравенства и значение правой части уже заданы. Для ЗСССН с фиксированным истинным значением $x^{0}$ число различных неравенств вида (1) равно $2^{n-1}$, так как этим неравенствам должен удовлетворять вектор $x^{0}$.

Случай $\mathcal{B}$ отличается от случая $\mathcal{A}$ двумя предположениями:

1) $m=n / 2$ и является нечетным числом;

2) $\sum_{j=1}^{n} a_{i j}=0, i=1,2, \ldots, t$.

Случай $\mathcal{B}$ проще анализировать, случай $\mathcal{A}$ при $n, t \rightarrow \infty, m \sim n / 2$ приближается по результатам анализа к случаю $\mathcal{B}$. Еще заметим, что в случае $\mathcal{B}$ коэффициенты в неравенствах вида (1) становятся зависимыми, и любая из плоскостей, задаваемых уравнением

$$
\sum_{j=1}^{n} a_{i j} x_{j}=0, \quad i=1,2, \ldots, t,
$$

строго делит объем единичного куба пополам и не содержит вершин куба, удовлетворяющих условию

$$
\sum_{j=1}^{n} x_{j}=m, \quad m-\text { нечетное число. }
$$

Поэтому каждому неравенству вида (1) удовлетворяет ровно половина из двоичных $n$-мерных векторов. 
Множество возможных решений состоит из векторов $\left(x_{1}, x_{2}, \ldots, x_{n}\right)$ веса $m$. Поэтому для случая В систему неравенств вида (1) можно, используя дополнительное уравнение

$$
x_{1}+x_{2}+\ldots+x_{n}=m
$$

преобразовать к виду

$$
b_{i 1} x_{1}+\ldots+b_{i n} x_{n} \geq(m+1) / 2, \quad i=1, \ldots, t,
$$

где

$$
b_{i 1}+\ldots+b_{i n}=m, \quad i=1, \ldots, t,
$$

коэффициенты в неравенстве (2) принимают значения 0 и 1. Для этого достаточно сложить каждое из неравенств (1) с приведенным выше равенством и результат сложения поделить на два.

Для $\mathrm{CCH}$ в случае $\mathcal{B}$ число неравенств вида (1) равно $C_{2 m}^{m}$, а для $3 \mathrm{CCCH}$ это число в два раза меньше.

\section{§3. Случайные системы неравенств}

Основной задачей для $\mathrm{CCH}$ в случае $\mathcal{B}$ является выяснение того, совместна система или нет. Ниже описываются алгебраические подходы к решению этой задачи. Анализ случая $\mathcal{A}$ приведен в [5]. В этой работе для $\mathrm{CCH}$ найдены условия несовместности системы, а для ЗСССН - апостериорные распределения неизвестных.

Сложив все $t_{1 i}$ неравенств из системы (2), в которых коэффициент при $x_{i}$ равен 1 , получим неравенство

$$
\begin{gathered}
t_{1 i} x_{i}+L_{1 i}\left(x_{1}, \ldots, x_{n}\right) \geq t_{1 i}(m+1) / 2 \\
L_{1 i}\left(x_{1}, \ldots, x_{n}\right)=c_{i 1} x_{1}+c_{i 2} x_{2}+\ldots+c_{i n} x_{n}, \quad c_{i i}=0, \quad i=1, \ldots, n .
\end{gathered}
$$

Так как

$$
b_{i 1}+b_{i 2}+\ldots+b_{i n}=m
$$

в неравенствах (2), то сумма коэффициентов в левой части неравенства (3) равна $t_{i 1} m$, а сумма коэффициентов в линейной форме $L_{1 i}\left(x_{1}, \ldots, x_{n}\right)$ равна $t_{1 i}(m-1)$.

Пусть $N_{1 i}(k)$ есть сумма $k$ наибольших коэффициентов из линейной формы $L_{1 i}\left(x_{1}, \ldots, x_{n}\right)$. Очевидно, что при выполнении условия

$$
t_{1 i}+N_{1 i}(m-1)<t_{1 i}(m+1) / 2
$$

неравенство (3) не имеет решений веса $m$, если выполняется равенство (4). 
Лемма 1. В случае $\mathcal{B}$ существуют такие числа $t_{1 i}, m$ и линейная форма $L_{1 i}$, что неравенство (3) не имеет решений.

Доказательство. Положим $t_{1 i}=2 m-1$, и пусть

$$
c_{i j}=\frac{t_{1 i}(m-1)}{2 m-1}=m-1 \quad \text { для всех } j \neq i .
$$

В этом случае

$$
t_{1 i}+N_{1 i}(m-1)=2 m-1+(m-1)^{2}=m^{2},
$$

а правая часть неравенства (3) равна

$$
\frac{t_{1 i}(m+1)}{2}=\frac{(2 m-1)(m+1)}{2}=m^{2}+\frac{m-1}{2}>m^{2} .
$$

Следовательно, рассматриваемое неравенство не имеет решений.

ПримеР 1. Пусть $n=10, m=5, t_{11}=9, c_{1 j}=4, j=2, \ldots, 9$. Тогда

$$
\begin{gathered}
t_{11}+N_{11}(4)=9+4 \cdot 4=25, \\
\frac{t_{11}(m+1)}{2}=9 \cdot \frac{6}{2}=27 .
\end{gathered}
$$

Это означает, что система (2) несовместима. Здесь возможен и другой вариант значений коэффициентов линейной формы $L_{11}$ :

$$
c_{12}=5, \quad c_{13}=c_{14}=\ldots=c_{19}=4, \quad c_{1,10}=3 .
$$

Если же

$$
N_{1 i}(m)<t_{1 i}(m+1) / 2, \mathrm{a} t_{1 i}+N_{1 i}(m-1) \geq t_{1 i}(m+1) / 2,
$$

то система (2) может иметь решения только при $x_{i}=1$. Для нашего примера это имеет место при

$$
c_{12}=c_{13}=6, \quad c_{14}=c_{15}=5, \quad c_{16}=c_{17}=4, \quad c_{18}=c_{19}=c_{1,10}=3 .
$$

Заметим, что аналогичные рассуждения можно провести и для новых неизвестных $y_{i}=1-x_{i}, i=1, \ldots, n$. В этом случае используются остальные $t_{0 i}$ неравенств из системы (1), $t_{0 i}+t_{1 i}=t$, и если $y_{i}=1$, то $x_{i}=0$. 
Укажем условия, при которых $x_{i}=0$. Сложив $t_{0 i}$ неравенств из системы (2), в которых коэффициент при $x_{i}$ равен нулю, придем к неравенству

$$
\begin{gathered}
L_{0 i}\left(x_{1}, \ldots, x_{n}\right) \geq t_{0 i}(m+1) / 2, \\
L_{0 i}\left(x_{1}, \ldots, x_{n}\right)=d_{i 1} x_{1}+\ldots+d_{i n} x_{n}, \quad d_{i i}=0 .
\end{gathered}
$$

Как и в предыдущем случае, обозначим через $N_{0 i}(k)$ сумму $k$ наибольших коэффициентов из линейной формы $L_{0 i}\left(x_{1}, \ldots, x_{n}\right)$. Тогда нетрудно заметить, что $x_{i}=0$ при выполнении следующих неравенств:

$$
N_{0 i}(m-1)<t_{0 i}(m+1) / 2, \quad N_{0 i}(m) \geq t_{0 i}(m+1) / 2 .
$$

Если же выполняется неравенство

$$
N_{0 i}(m)<t_{0 i}(m+1) / 2,
$$

то система (2) несовместна.

Система неравенств (2) несовместна, если из $t_{1 i}$ неравенств следует, что $x_{i}=1$, а из $t_{0 i}$ других неравенств - что $x_{i}=0$. Приведем условия, когда это осуществляется. Если одновременно выполняются четыре неравенства

$$
\begin{gathered}
N_{1 i}(m)<t_{1 i}(m+1) / 2, \\
t_{1 i}+N_{1 i}(m-1) \geq t_{1 i}(m+1) / 2, \\
N_{0 i}(m-1)<t_{0 i}(m+1) / 2, \\
N_{0 i}(m) \geq t_{0 i}(m+1) / 2,
\end{gathered}
$$

то система (2) не имеет решений.

Дополнительно заметим, что помимо определения значения $x_{i}$ мы сможем в ряде случаев определить значения и других неизвестных. Для иллюстрации этой возможности приведем пример.

ПримеР 2. Пусть $n=10, m=5, t_{11}=12, N_{11}(k)=b_{2}+b_{3}+\ldots+b_{k+1}$. Неравенство (3) имеет вид

$$
\begin{gathered}
12 x_{1}+b_{2} x_{2}+\ldots+b_{10} x_{10} \geq 36 \\
b_{2}+b_{3}+\ldots+b_{10}=48, \quad b_{2} \geq b_{3} \geq \ldots \geq b_{10} .
\end{gathered}
$$

Предположим, что $b_{2}+b_{3}+b_{4}+b_{5}=N_{11}(4)=24$. Тогда для «среднего» варианта $\left(b_{2}, b_{3}, \ldots, b_{10}\right)=(6,6,6,6,5,5,5,5,4)$ находим значения всех неизвестных $x_{1}=x_{2}=x_{3}=x_{4}=x_{5}=1, x_{6}=x_{7}=x_{8}=x_{9}=x_{10}=0$. Если же $\left(b_{2}, b_{3}, \ldots, b_{10}\right)=(7,7,5,5,5,5,5,5,4)$, то

$$
x_{1}=x_{2}=x_{3}=1, \quad x_{10}=0, \quad x_{4}+x_{5}+x_{6}+x_{7}+x_{8}+x_{9}=1 .
$$


Сформулируем полученные выше результаты в следующем утверждении.

Теорема 1. Система неравенств (2) несовместна в следующих случаях:

1) если найдется хотя бы одно $i \in\{1,2, \ldots, n\}$, для которого

$$
t_{1 i}+N_{1 i}(m-1)<t_{1 i}(m+1) / 2 \quad \text { или } \quad N_{0 i}(m)<t_{0 i}(m+1) / 2 ;
$$

2) если найдется хотя бы одно $i \in\{1,2, \ldots, n\}$, для которого одновременно выполняются неравенства

$$
\begin{gathered}
N_{1 i}(m)<t_{1 i}(m+1) / 2, \\
t_{1 i}+N_{1 i}(m-1) \geq t_{1 i}(m+1) / 2, \\
N_{0 i}(m-1)<t_{0 i}(m+1) / 2, \\
N_{0 i}(m) \geq t_{0 i}(m+1) / 2 .
\end{gathered}
$$

Если на первом этапе мы не определили значение $x_{1}$, то переходим ко второму этапу - определению значения $x_{2}$, и т. д. Найдя значение некоторого неизвестного, подставляем это значение в исходную систему неравенств и продолжаем процесс с уже меньшим числом неизвестных. В итоге мы либо найдем полностью или частично решение исходной системы неравенств, либо определим ее несовместность при условии на значения неизвестных

$$
x_{1}+x_{2}+\ldots+x_{n}=m \text {. }
$$

Здесь следует заметить, что после подстановки в систему неравенств найденного значения одного неизвестного может измениться вид исходной системы. В частности, если $x_{1}=0$, то сокращается число неизвестных, но правая часть неравенств не меняется. Для $x_{1}=1$ в неравенствах, в которых присутствует $x_{1}$, не меняется правая часть, равная $(m+1) / 2$, а в остальных неравенствах правая часть заменяется на $(m-1) / 2$. При этом дополнительно становится известно, что

$$
x_{2}+\ldots+x_{n}=m-1 .
$$

В случае затруднений нахождения значений отдельных неизвестных можно перейти к оценке значений некоторых специально подобранных линейных форм от небольшого числа неизвестных. Рассмотрим общий случай: линейное псевдобулево неравенство

$$
c_{1} y_{1}+c_{2} y_{2}+\ldots+c_{n_{1}} y_{n_{1}} \geq C,
$$

2012. T. 3. № 3. C. 5-19 
в котором все коэффициенты целые числа, а

$$
\begin{gathered}
c_{1}=c_{2}>c_{3} \geq c_{4} \geq \ldots \geq c_{n_{1}}, \\
y_{1}+y_{2}+\ldots+y_{n_{1}}=m_{1}, \quad 3 \leq m_{1}<n_{1} .
\end{gathered}
$$

Имеют место следующие факты:

1) $y_{1}=y_{2}=1$, если выполняются неравенства

$$
C-2 c_{1} \leq \sum_{i=3}^{m_{1}} c_{i}<C-c_{1}-c_{m_{1}+1} ;
$$

2) $y_{1}+y_{2} \geq 1$, если справедливо неравенство

$$
\sum_{i=3}^{m_{1}+2} c_{i}<C
$$

3) $y_{j}=y_{j+1}=\ldots=y_{n_{1}}=0$ для $j \in\left[m_{1}+1, m_{1}+2, \ldots, n_{1}\right], c_{j}<c_{m_{1}}$, если

$$
2 c_{1}+\sum_{i=3}^{m_{1}-1} c_{i}+c_{j}<C .
$$

Если система неравенств несовместна, то это означает, что либо перед нами $\mathrm{CCH}$, либо ЗСССН с $\left|x^{0}\right| \neq m$. В любом случае следует повторить описанный процесс с другим значением $\left|x^{0}\right|$. Для ЗСССН мы сможем найти ограничения на значение $\left|x^{0}\right|$. Максимальная трудоемкость этого алгоритма в элементарных операциях пропорциональна $t n^{3}$.

\section{§4. Заведомо совместные системы неравенств с возможными искажениями в знаках неравенств}

Рассмотрим случай $\mathcal{B}$. Предположим, что система неравенств вида (1) заведомо совместна с истинным решением $x^{0}$ веса $m, m-$ нечетное число, $n=2 m$. Построим новую случайную систему, изменяя знак неравенства в $j$-м неравенстве из этой системы с вероятностью $p$ на обратный. В этом случае $j$-е неравенство имеет вид

$$
-a_{j 1} x_{1}-\ldots-a_{j n} x_{n} \geq 1
$$

и ему не удовлетворяет решение $x^{0}$. С вероятностью $1-p$ знак неравенства не меняется. 
Такая ситуация возникает при анализе булевых систем уравнений

$$
\varphi_{i}(\bar{x})=b_{i}, \quad i=1,2, \ldots, t,
$$

с пороговыми функциями в левой части из класса $\mathcal{K}$ и с искаженной правой частью. Если $b_{i}=\varphi_{i}\left(\bar{x}^{0}\right)$, то уравнение заменяется неравенством $\left(\bar{a}_{i}, \bar{x}\right) \geq 1$. Если же $b_{i} \neq \varphi_{i}\left(\bar{x}^{0}\right)$, то это уравнение заменяется неравенством $\left(\bar{a}_{i}, \bar{x}\right) \leq-1$, и будем говорить, что в этом случае произошла ошибка. Аналогично выглядит и вариант замены произвольной функции $\varphi_{i}(\bar{x})$ на псевдобулев статистический аналог - линейное псевдобулево неравенство (пороговую функцию из класса $\mathcal{K})$.

Рассмотрим два неравенства

$$
(\bar{c}, x) \geq 1, \quad(\bar{b}, x) \geq 1
$$

и найдем

$$
\mathbf{P}\left\{\left(\bar{c}+\bar{b}, x^{0}\right) \geq 2\right\} .
$$

Нетрудно видеть, что в нашем случае для независимых и одинаково распределенных векторов $\bar{c}$ и $\bar{b}$ величина

$$
\mathbf{P}\left\{\left(\bar{c}+\bar{b}, x^{0}\right) \geq 2\right\}=(1-p)^{2}+p(1-p) \cdot\left[1-\sum_{i \geq 0} p_{2 i+1}^{2}\right],
$$

где

$$
p_{2 i+1}=\mathbf{P}\left\{\left(\bar{c}, x^{0}\right)=2 i+1 \mid\left(\bar{c}, x^{0}\right) \geq 1\right\}, \quad i \geq 0 .
$$

Лемма 2. Справедливы равенства

$$
p_{2 i+1}=2 \cdot\left(C_{m}^{k+i}\right)^{2} / C_{2 m}^{m}, k=\frac{m+1}{2}, \quad i \geq 0 .
$$

Доказательство. Не теряя общности, положим $x_{1}^{0}=x_{2}^{0}=\ldots=x_{m}^{0}=1$. Тогда

$$
\begin{gathered}
\mathbf{P}\left\{\left(\bar{c}, x^{0}\right)=2 i+1 \mid\left(\bar{c}, x^{0}\right) \geq 1\right\}= \\
=\mathbf{P}\left\{c_{1}+c_{2}+\ldots+c_{m}=2 i+1 \mid c_{1}+c_{2}+\ldots+c_{m} \geq 1\right\}=2 \cdot\left(C_{m}^{k+i}\right)^{2} / C_{2 m}^{m} .
\end{gathered}
$$

При этом выполняется равенство

$$
\sum_{i \geq 0} p_{2 i+1}=1
$$

2012. T. 3. № 3. C. 5-19 
так как

$$
\sum_{j \geq 0}\left(C_{m}^{j}\right)^{2}=C_{2 m}^{m}, \quad \sum_{i \geq 0}\left(C_{m}^{k+i}\right)^{2}=\sum_{j \geq 1}\left(C_{m}^{k-j}\right)^{2}
$$

Следствие. При $m \rightarrow \infty$

$$
\sum_{i \geq 0} p_{2 i+1}^{2} \rightarrow 0, \quad \mathbf{P}\left\{\left(\bar{c}+\bar{b}, x^{0}\right) \geq 2\right\} \rightarrow 1-p
$$

В лемме 4 работы [5] похожим способом (но для равновероятного случая) доказано, что вероятность того, что истинное решение $x^{0}$ не удовлетворяет неравенству

$$
\left(\bar{a}_{i}+\bar{a}_{j}, x^{0}\right) \geq 2
$$

равна выражению

$$
p \cdot\left[1+(1-p) \cdot C_{2 m}^{m} / 2^{2 m-1}\right]
$$

Это говорит о том, что при сложении двух неравенств в общем случае вероятность ошибки (невыполнения суммарного неравенства для истинного решения $x^{0}$ ) существенно не меняется, но становится больше.

Пусть случайные величины $\eta_{1}, \ldots, \eta_{t}$ независимы и одинаково распределены:

$$
\begin{gathered}
\mathbf{P}\left(\eta_{j}=2 i+1\right)=p_{2 i+1}=\mathbf{P}\left\{\left(\bar{a}_{j}, x^{0}\right)=2 i+1 \mid\left(\bar{a}_{j}, x^{0}\right) \geq 1\right\}, \\
i \geq 0, j=1,2, \ldots, t .
\end{gathered}
$$

Рассмотрим случайную величину

$$
\eta=\varepsilon_{1} \eta_{1}+\varepsilon_{2} \eta_{2}+\ldots+\varepsilon_{t} \eta_{t}
$$

Здесь $\varepsilon_{1}, \varepsilon_{2}, \ldots, \varepsilon_{t}-$ независимые и одинаково распределенные случайные величины,

$$
\mathbf{P}\left(\varepsilon_{j}=1\right)=1-p, \quad \mathbf{P}\left(\varepsilon_{j}=-1\right)=p, \quad j=1,2, \ldots, t .
$$

Очевидно, что в нашем случае для системы (1) при наличии искажений в знаках неравенств

$$
\eta=\sum_{i=1}^{t}\left(\bar{a}_{i}, x^{0}\right)
$$


Сложив все $t$ неравенств вида (1), получим неравенство

$$
\sum_{i=1}^{t}\left(\bar{a}_{i}, x\right) \geq t .
$$

Следовательно, вероятность того, что истинное решение $x^{0}$ удовлетворяет неравенству (5), равна $\mathbf{P}(\eta \geq t)$.

При $t \rightarrow \infty$ распределение случайной величины $\xi=\frac{\eta-\mathbf{M} \eta}{\sqrt{\mathbf{D} \eta}}$ стремится к стандартному нормальному распределению $N(0,1)$,

$$
\mathbf{M} \eta=t \cdot(1-2 p) \cdot \mathbf{M} \eta_{1}, \quad \mathbf{D} \eta=t \cdot \mathbf{D}\left(\varepsilon_{1} \eta_{1}\right) .
$$

Находим:

$$
\begin{aligned}
\mathbf{D}\left(\varepsilon_{1} \eta_{1}\right) & =\mathbf{M}\left(\varepsilon_{1} \eta_{1}-\mathbf{M} \varepsilon_{1} \eta_{1}\right)^{2}=\mathbf{M} \eta_{1}^{2}-(1-2 p)^{2}\left(\mathbf{M} \eta_{1}\right)^{2}, \\
\mathbf{M} \eta_{1} & =\sum_{i \geq 0}(2 i+1) \cdot p_{2 i+1}=1+4 \sum_{i \geq 0} i\left(C_{m}^{k+i}\right)^{2} / C_{2 m}^{m} .
\end{aligned}
$$

Если $p$ и $\mathbf{M} \eta_{1}$ таковы, что

$$
(1-2 p) \cdot \mathbf{M} \eta_{1}=1+L, \quad L>\varepsilon>0,
$$

то при больших $t$ можно воспользоваться приближением

$$
\begin{gathered}
\mathbf{P}\left\{\sum_{i=1}^{t}\left(a_{i}, x^{0}\right) \geq t\right\}=\mathbf{P}\left\{\xi \geq \frac{t-\boldsymbol{M} \eta}{\sqrt{\mathbf{D} \eta}}\right\} \approx \frac{1}{\sqrt{2 \pi}} \int_{\theta}^{\infty} e^{-z^{2} / 2} d z, \\
\theta=t \cdot L / \sqrt{t \cdot \mathbf{D}\left(\varepsilon_{1} \eta_{1}\right)}=L \sqrt{t / \mathbf{D}\left(\varepsilon_{1} \eta_{1}\right)} .
\end{gathered}
$$

Заметим, что при наличии ограничения на истинное решение

$$
x_{1}^{0}+x_{2}^{0}+\ldots+x_{n}^{0}=m
$$

где $m=n / 2$ и является нечетным числом, возможно, удобнее пользоваться другой записью неравенств системы (1), а именно

$$
\left(\bar{a}_{i}, x\right)>0 \quad \text { или } \quad\left(\bar{a}_{i}, x\right)<0,
$$

где $x=\left(x_{1}, x_{2}, \ldots, x_{n}\right), a_{i}=\left(a_{i 1}, a_{i 2}, \ldots, a_{i n}\right), i=1,2, \ldots t$.

Действительно, исходное булево уравнение $\varphi_{i}(x)=b_{i}$ мы заменяем на аналог - линейное псевдобулево неравенство приведенного вида, и при 
этом вероятность ошибки равна $p$. Это означает, что истинное решение $x^{0}$ удовлетворяет неравенству с вероятностью $1-p$. Дополнительно напомним, что истинное решение $x^{0}$ не удовлетворяет равенствам

$$
\left(\bar{a}_{i}, x^{0}\right)=0, \quad i=1,2, \ldots, t .
$$

Итак, перед нами $t_{1}$ неравенств

$$
\left(\bar{a}_{i}, x\right)>0, \quad i=1,2, \ldots, t_{1}
$$

и $t-t_{1}=t_{0}$ неравенств

$$
\left(\bar{a}_{j}, x\right)<0, \quad j=t_{1}+1, t_{1}+2, \ldots, t .
$$

Каждое неравенство сложим почленно с ограничением на значения неизвестных

$$
x_{1}+x_{2}+\ldots+x_{n}=m
$$

и поделим полученные соотношения на два. В итоге придем к системе неравенств

$$
\begin{array}{ll}
b_{i 1} x_{1}+\ldots+b_{i n} x_{n}>m / 2, & i=1,2, \ldots, t_{1} \\
b_{j 1} x_{1}+\ldots+b_{j n} x_{n}<m / 2, & j=t_{1}+1, t_{1}+2, \ldots, t
\end{array}
$$

Во всех неравенствах

$$
b_{k 1}+\ldots+b_{k n}=m, \quad b_{k l} \in\{0,1\}, \quad k=1,2, \ldots, t, \quad l=1,2, \ldots, n .
$$

Алгебраические методы нахождения значений отдельных неизвестных, изложенные в п. 3 для ССН, для ЗСССН с искаженными знаками неравенств не эффективны, так как число неравенств $t$, необходимое для анализа системы (6), существенно больше числа неравенств, необходимого для обнаружения факта несовместности ССН. Поэтому мы попробуем оценить апостериорное распределение отдельных неизвестных на основе информации, содержащейся в системе (6).

Предположим, что $x_{i}^{0}=1$, и найдем вероятность появления неравенства $\left(\bar{b}_{1}, x\right)>m / 2$ с $b_{1 i}=1$. Легко видеть, что в силу равновероятности распределения коэффициентов в неравенствах исходной системы

$$
\begin{aligned}
\mathbf{P}\left\{\left(\overline{b_{1}}, x\right)>m / 2\right\} & =\mathbf{P}\left\{\left(\overline{b_{1}}, x\right)<m / 2\right\}=1 / 2, \\
\mathbf{P}\left\{b_{1 i}=1\right\} & =\mathbf{P}\left\{b_{1 i}=0\right\}=1 / 2 .
\end{aligned}
$$


Поэтому

$$
\begin{gathered}
\mathbf{P}\left\{\left(\overline{b_{1}}, x\right) \geq(m+1) / 2 \mid x_{i}^{0}=1, b_{1 i}=1\right\}= \\
=(1-p) \mathbf{P}\left\{\left(\overline{b_{1}}, x^{0}\right) \geq \frac{m+1}{2} \mid x_{i}^{0}=1, b_{1 i}=1\right\}+ \\
+p \mathbf{P}\left\{\left(\overline{b_{1}}, x^{0}\right) \leq \frac{m-1}{2} \mid x_{i}^{0}=1, b_{1 i}=1\right\}= \\
=(1-p) \sum_{j \geq 0} C_{m-1}^{j+(m-1) / 2} C_{n-m}^{-j+(m-1) / 2} / C_{n-1}^{m-1}+p \sum_{j \geq 0} C_{m-1}^{-j+(m-3) / 2} C_{n-m}^{j+(m+1) / 2} / C_{n-1}^{m-1} .
\end{gathered}
$$

Для нашего примера из п. 3 при $n=10, m=5$ искомая вероятность равна

$$
\frac{1}{2} \cdot\left[1+(1-2 p) \cdot \frac{C_{4}^{2} C_{5}^{3}}{C_{9}^{4}}\right]=\frac{1}{2} \cdot\left[1+\frac{(1-2 p) 10}{21}\right]>\frac{1}{2} .
$$

Если искажения в знаках неравенств отсутствуют, т. е. $p=0$, тогда эта вероятность равна $31 / 42 \approx 3 / 4$. Для ССН эта вероятность равна $1 / 2$. Этот факт можно использовать при построении критерия различия $\mathrm{CCH}$ и $3 \mathrm{CCCH}$.

Пусть $x_{i}^{0}=0$, тогда

$$
\begin{gathered}
\mathbf{P}\left\{\left(\overline{b_{1}}, x\right) \geq(m+1) / 2 \mid x_{i}^{0}=0, b_{1 i}=0\right\}= \\
=(1-p) \sum_{j \geq 0} C_{m}^{j+(m+1) / 2} C_{m-1}^{-j+(m-1) / 2} / C_{2 m-1}^{m}+p \sum_{j \geq 0} C_{m}^{-j+(m-1) / 2} C_{m-1}^{j+(m+1) / 2} / C_{2 m-1}^{m} .
\end{gathered}
$$

Заметим, что обе приведенные вероятности равны, так как $n=2 m$ и под знаками сумм у них переставлены одинаковые сомножители.

Лемма 3. Вероятность появления неравенства $\left(\bar{b}_{1}, x\right)>m / 2$ в системе (6) при условии, что $x_{i}^{0}=1, b_{i 1}=1$ или $x_{i}^{0}=0, b_{i 1}=0$, равна

$$
\frac{1}{2}\left[1+(1-2 p) \cdot C_{m-1}^{(m-1) / 2} \cdot C_{m}^{(m-1) / 2} / C_{2 m-1}^{m-1}\right] .
$$

Доказательство. Очевидно, что

$$
\begin{aligned}
& \operatorname{coef}_{x}\left(1+\frac{1}{x}\right)^{m-1} \cdot(1+x)^{m}=C_{2 m-1}^{m-1}=\sum_{i \geq 0} C_{m-1}^{i} C_{m}^{i+1}= \\
= & \sum_{i=0}^{(m-3) / 2} C_{m-1}^{i} C_{m}^{i+1}+C_{m-1}^{(m-1) / 2} C_{m}^{(m+1) / 2}+\sum_{j=(m+1) / 2}^{m-1} C_{m-1}^{j} C_{m}^{j+1} .
\end{aligned}
$$

2012. T. 3. № 3. C. 5-19 
В этом выражении первое и третье слагаемые равны и совпадают со второй суммой из формулы (7). Сумма первого и второго слагаемых равна первой сумме из формулы (7). Используя эти факты, приходим к утверждению леммы.

В работе [5] приведены апостериорные вероятности значений координат истинного решения $x^{0}$ для случая $\mathcal{A}$. Случай $\mathcal{B}$ рассматривается аналогичным образом с использованием формулы (8).

В заключение определим среднее число самых близких к истинному решений, отличающихся от истинного в двух координатах и удовлетворяющих системе неравенств (6) при отсутствии искажений.

Не теряя общности, положим

$$
x_{1}^{0}=x_{2}^{0}=\ldots=x_{m}^{0}=1, x_{m+1}^{0}=x_{m+2}^{0}=\ldots=x_{2 m}^{0}=0
$$

и найдем условную вероятность

$$
\mathbf{P}\left\{\left(\overline{b_{1}}, x^{0} \oplus e_{1,2 m}\right) \geq(m+1) / 2 \mid\left(\overline{b_{1}}, x^{0}\right) \geq(m+1) / 2\right\}
$$

для вектора $e_{1,2 m}=(1,0, \ldots, 0,1),\left|e_{1,2 m}\right|=2$. Очевидно, что неравенство

$$
b_{12}+\ldots+b_{1 m}+b_{1,2 m} \geq(m+1) / 2
$$

выполняется при условии

$$
b_{11}+b_{12}+\ldots+b_{1 m} \geq(m+1) / 2
$$

только в следующих случаях:

$$
\begin{aligned}
& \text { 1. } b_{12}+\ldots+b_{1 m} \geq(m+1) / 2, \\
& \text { 2. } b_{11}=1, b_{12}+\ldots+b_{1 m}=(m-1) / 2, b_{1,2 m}=1 .
\end{aligned}
$$

Указанное неравенство не выполняется только при

$$
b_{11}=1, \quad b_{12}+\ldots+b_{1 m}=(m-1) / 2, \quad b_{1,2 m}=0 .
$$

Вероятность этого события оценивается выражением

$$
p(2)=2\left(C_{m-1}^{(m-1) / 2}\right)^{2} / C_{2 m}^{m}=\frac{4}{\sqrt{\pi m}}\left[1+O\left(\frac{1}{m}\right)\right], \quad m \rightarrow \infty .
$$

Для противоположного неравенства условная вероятность

$$
1-p(2)=\mathbf{P}\left\{\left(\bar{b}_{1}, x^{0} \oplus e_{1,2 m}\right) \leq(m-1) / 2 \mid\left(\bar{b}_{1}, x^{0}\right) \leq(m-1) / 2\right\}
$$


будет такая же, как и приведенная выше. Поэтому с вероятностью

$$
[1-p(2)]^{t}=\exp \left\{-t\left[\frac{4}{\sqrt{\pi m}}+O\left(\frac{1}{m}\right)\right]\right\}
$$

при $m \rightarrow \infty$ вектор $x^{0} \oplus e_{1,2 m}$ будет удовлетворять исходной системе неравенств (1), а среднее число $N(2)$ самых близких к истинному решений выражается формулой

$$
N(2)=m^{2}[1-p(2)]^{t}=\exp \left\{2 \ln m-\frac{4 t}{\sqrt{\pi m}}+O\left(\frac{t}{m}\right)\right\} .
$$

Отсюда приходим к следующему утверждению.

Теорема 2. Пусть $4 t / \sqrt{\pi m}=(2+\varepsilon) \ln m$. Тогда $N(2) \rightarrow \infty$ при $m \rightarrow$ $\rightarrow \infty$, если $\varepsilon<0, и N(2) \rightarrow 0$ при $m \rightarrow \infty$ для $\varepsilon>0$.

\section{Список литературы}

1. Балакин Г.В., Никонов В.Г. Методы сведения булевых уравнений к системам пороговых соотношений // Обозр. прикл. и промышл. матем. 1994. - Т. 1. Вып. 3. - С. 389-401.

2. Никонов В.Г. Пороговые представления булевых функций // Обозр. прикл. и промышл. матем. - 1994. - Т. 1. Вып. 3. - С. 402-457.

3. Балакин Г.В. Об одном свойстве линейных целочисленных неравенств с искаженной правой частью // Обозр. прикл. и промышл. матем. 2007. - Т. 14. Вып. 6. - С. 1071-1072.

4. Балакин Г.В. Об одной линейной псевдобулевой системе неравенств // Обозр. прикл. и промышл. матем. - 2008. - Т. 15. Вып. 4. - С. 738.

5. Балакин Г.В. Линейные псевдобулевы неравенства // Математические вопросы криптографии. - 2010. - Т. 1. Вып. 3. - С. 5-18. 